\title{
CONVERGENT ANALYTICAL TOOLS FOR BIG DATA APPLICATIONS IN HADOOP ENVIRONMENT
}

\author{
Bhagyashree A V \\ M.Tech Scholar, \\ Dept. Of. Computer Science \& Engineering, \\ BMS Institute of Technology \& Management, \\ Yelahanka, Bengaluru
}

\begin{abstract}
Big Data Analytics offers an about interminable wellspring of business and instructive understanding, that can prompt operational improvement and new open doors for organizations to give undiscovered income crosswise over pretty much every industry. From use cases like client personalization, to hazard relief, to misrepresentation discovery, to inward tasks investigation, and the various new use cases emerging close every day, the value covered up in organization information has organizations hoping to make a front-line examination activity. Finding an incentive inside crude information presents numerous difficulties for IT groups. Each organization has various needs and various information resources. Business activities change rapidly in a regularly quickening commercial center, and staying aware of new orders can require readiness and versatility. In addition, a fruitful Big Data Analytics activity requires tremendous figuring assets, innovative framework, and exceptionally talented faculty. These troubles can make various exercises flop before they pass on regard. Beforehand, a nonappearance of enlisting power and access to computerization made a certified age scale examination movement past the compass of most associations: Big Data was too much expensive, with a ton of issue, and no sensible ROI. With the climb of conveyed registering and new headways in figure resource the load up, Big Data gadgets are more accessible than some other time in ongoing memory.
\end{abstract}

Keywords- Big data, analytical tools, Apache Hadoop, Storm, Hive, Pig

\section{INTRODUCTION}

Big data implies the datasets which can't be perceived, acquired, oversaw, examined, and handled by present devices. Various meanings of huge information have been given by various clients of Big Data and various experts of Big Data like research researchers, information examiners, and specialized professionals. Big Data Analysis essentially includes logical techniques for huge information, precise

\author{
Anjan K Koundinya \\ Associate Professor and PG Coordinator, \\ Dept. Of. Computer Science \& Engineering, \\ BMS Institute of Technology \& Management, \\ Yelahanka, Bengaluru
}

engineering of huge information, and huge information digging and programming for examination. Information examination is the most significant advance in Big Data, for investigating important qualities, giving recommendations and choices. Potential qualities can be investigated by information examination. In any case, investigation of information is a wide region, which is dynamic and is mind boggling.

\section{BIG DATA ANALYSIS}

Big data examination alludes to the technique of dissecting enormous volumes of information, or huge information. This huge information is accumulated from a wide assortment of sources, including interpersonal organizations, recordings, computerized pictures, sensors, and deals exchange records. The point in examining this information is to reveal examples and associations that may some way or another be undetectable, and that may give significant bits of knowledge about the clients who made it. Through this knowledge, organizations might have the option to increase an edge over their adversaries and settle on prevalent business choices.

Modern programming projects are utilized for big data investigation, yet the unstructured information utilized in huge information examination may not be appropriate to ordinary information distribution centers. Big data's high preparing prerequisites may likewise make customary information warehousing a poor fit. Accordingly, more up to date, greater information investigation conditions and advancements have raised, including Hadoop, Map Reduce and Cassandra (Nosql) databases. These innovations make up an open-source programming structure that is utilized to process gigantic informational collections over grouped frameworks.

\section{ANALYTICAL TOOLS OF BIG DATA}

\subsection{Apache Hadoop:}

The Apache Hadoop programming library is a system that takes into consideration the appropriated preparing of enormous informational collections crosswise over bunches of 


\section{International Journal of Engineering Applied Sciences and Technology, 2020 Vol. 4, Issue 9, ISSN No. 2455-2143, Pages 283-285 \\ Published Online January 2020 in IJEAST (http://www.ijeast.com)}

PCs utilizing basic programming models. It is intended to scale up from single servers to a large number of machines, each offering nearby calculation and capacity. As opposed to depend on equipment to convey high-accessibility, the library itself is intended to recognize and deal with disappointments at the application layer, so conveying an exceptionally accessible administration over a group of PCs, every one of which might be inclined to disappointments.

Data analysis using hadoop MapReduce environment manages investigation of YouTube information utilizing Hadoop MapReduce structure on a cloud stage AWS [1]. Hadoop multi node bunch is setup on private cloud which is AWS (Amazon Web Services). Inside AWS, they set up EC2 occurrences with a name node and five information nodes. The video measurements got from the API are put away into the Hadoop Distributed File System and the information preparing is finished by the MapReduce framework. The YouTube informational collection is dissected utilizing MapReduce Algorithm to find beneath measurements:

- The Top most 5 categories in which most number of videos are uploaded

- The Top 5 up loaders

- The Top 5 most viewed videos

This information empowers us to examine interests of individuals through long range informal communication gathering. This encourages in settling on choices to put resources into all the more drifting territories to profit individuals.

Due of its particular nature of Big Data, it is put away in disseminated document framework models. Hadoop and HDFS by Apache are generally utilized for putting away and overseeing Big Data. Analyzing Big Data is a difficult assignment as it includes enormous dispersed record frameworks which ought to be deficiency tolerant, adaptable and versatile. Map Reduce is broadly been utilized for the proficient examination of Big Data. In a paper[2] they recommend various strategies for taking into consideration the issues close by through Map Reduce structure over Hadoop Distributed File System (HDFS). Map Reduce is a minimization system which utilizes record ordering with mapping, arranging, rearranging. Map Reduce methods have been contemplated in this paper which is executed for Big Data examination utilizing HDFS. Map Reduce employments utilize effective information preparing systems which can be applied in every one of the periods of mapReduce; to be specific Mapping, Shuffling, Combining, Grouping, Indexing and Reducing.

\subsection{Apache Spark:}

Spark have picked up a ton of footing over the previous decades and have progressed toward becoming hugely famous, particularly in ventures. It is ending up progressively obvious that compelling enormous information examination is critical to tackling man-made brainpower issues. Along these lines, a multi-calculation library was actualized in the Spark system, called MLlib. In a paper[3], they propose a novel structure that consolidates the distributive computational capacities of Apache Spark what's more, the propelled AI design of a profound multilayer perceptron (MLP), utilizing the mainstream idea of Cascade Learning. We direct experimental examination of our system on two genuine world datasets. The outcomes are empowering and validate our proposed system, thus demonstrating that it is an improvement over customary huge information examination techniques that use either Deep learning or Spark as individual components.

\subsection{Apache Storm:}

As of late Big Data has turned out to be perhaps the most sweltering subject in programming designing. Need to manage tremendous measure of information brings new difficulties and, obviously, new chances. Enormous Data requires modifying ways to deal with structuring programming design. In particular, the Lambda Architecture pattern distinguishes the components that process recent data only in real-time ("speed layer"). Practically speaking, such information things come constantly from supposed information streams. The point of a paper [4] is to speak to idea of the executed by the creators Apache Storm based topology for constant handling of information streams from interpersonal organizations. When all is said in done, considering the way that the measure of information is tremendous, it appears to be hard to process the information by and large. That is the reason ongoing information stream preparing has been a significant logical and building task for over 10 years. Generally, this errand contains two fixings: algorithms (that are responsible for acquiring investigative data) and software (that actualizes preparing framework).

The paper is dedicated to the subsequent fixing.

\subsection{Apache Pig:}

Pig Latin is actualized on Pig which is open source programming which kept running on Hadoop. Pig Latin's fundamental highlights incorporate help for a versatile settled information model, broad help for client characterized capacities, and the capacity to work on information records with no composition data. Pig Latin additionally accompanies a novel troubleshooting condition that is especially helpful when managing enormous informational indexes. In request to adequately deal with the developing sum of accessible RDF information, a versatile and adaptable RDF information preparing structure is required. A paper [5] recently proposed 


\section{International Journal of Engineering Applied Sciences and Technology, 2020 Vol. 4, Issue 9, ISSN No. 2455-2143, Pages 283-285 \\ Published Online January 2020 in IJEAST (http://www.ijeast.com)}

a Hadoop based system, which takes favorable circumstances of adaptable and fault tolerant disseminated handling advances, initially proposed as Google's dispersed record framework and MapReduce parallel model. In the paper, they present a technique broadening the Pig information preparing stage over the Hadoop framework. Pig assembles projects written in an abnormal state language, called Pig Latin, into MapReduce programs that can be executed by Hadoop. So as to help RDF, Pig was reached out with the capacity to load and store RDF information effectively. Moreover, as thinking is a significant prerequisite for most frameworks putting away RDF information, support for inducing new triples utilizing entailment standards was likewise included. In this paper, we depict these augmentations what's more; present an assessment of their exhibition.

\subsection{Apache Cassandra:}

Apache Cassandra is a main disseminated database of decision with regards to huge information the executives with zero personal time, straight adaptability, and consistent various server farm organization. With progressively more extensive selection of Cassandra for online exchange preparing by several Web-scale organizations, there is a developing requirement for a thorough and reasonable information displaying approach that guarantees sound and proficient diagram structure. A paper [6] work I) proposes the principal inquiry driven enormous information displaying approach for Apache Cassandra, ii) characterizes significant information displaying standards, mapping principles, and mapping designs to control sensible information demonstrating, iii) presents visual charts for Cassandra legitimate and physical information models, and iv) illustrates an information displaying device that computerizes the whole information demonstrating process. capacity to load and store RDF information productively. Besides, as thinking is a significant necessity for most frameworks putting away RDF information, support for deriving new triples utilizing entailment principles was likewise included. In this paper, we depict these expansions what's more, present an assessment of their exhibition.

\section{CONCLUSION}

There are numerous answers for location huge information systematic necessities. Most enormous information design arrangements use couple of apparatuses to assemble a total arrangement; this can help meet the stringent business necessities inside the most cost- enhanced, execution, and flexible way conceivable. The final product is a flexible, gigantic data engineering this can scale related to your business on the overall foundation.

\section{REFERENCES}

[1] PrathyushaRani Merla \& Yiheng Liang, "Data analysis sing Hadoop MapReduce environment”, 2017 IEEE International Conference on Big Data (Big Data)

[2] Shankar Ganesh Manikandan \& Siddarth Ravi, "Big Data Analysis Using Apache Hadoop", 2014 International Conference on IT Convergence and Security (ICITCS)

[3] Anand Gupta; Hardeo Kumar Thakur;Ritvik Shrivastava; Pulkit Kumar \& Sreyashi Nag, "A Big Data Analysis Framework Using Apache Spark and Deep Learning ”, 2017 IEEE International Conference on Data Mining Workshops (ICDMW)

[4] Anatoliy Batyuk; Volodymyr Voityshyn, "Apache Storm Based on Topology for Real-Time Processing of Streaming Data from Social Networks", 2018 IEEE 13th International Scientific and Technical Conference on Computer Sciences and Information Technologies (CSIT)

[5] Yusuke Tanimura, Akiyoshi Matono, Steven Lynden, Isao Kojima, "Extensions to the Pig data processing platform for scalable RDF data processing using Hadoop", 2010 IEEE 26th International Conference on Data Engineering Workshops (ICDEW 2010)

[6] Artem Chebotko; Andrey Kashlev; Shiyong Lu, "A Big Data Modeling Methodology for Apache Cassandra", Published in IEEE International Congress on Big Data 2015

[7] An article on Big Data analytics research paper at URLhttps://www.researchgate.net/publication/322629223_Big _Data_Analytics

[8] An article on Big Data analytics at URL https://www.techopedia.com/definition/28659/big$\underline{\text { dataanalytics }}$

[9]An article on Big Data analytics at URL https://searchbusinessanalytics.techtarget.com/definition/bigda $\underline{\text { ta-analytics }}$

[10] Syeda Sana Bukhari ; JinHyuck Park ; Dong Ryeol Shin, "Hadoop based Demography Big Data Management System", 2018 19th IEEE/ACIS International Conference on Software Engineering, Artificial Intelligence, Networking and Parallel/Distributed Computing (SNPD) 